\title{
Simulations of High Non-Uniform Electric Field in Dielectric Barrier Electrode System
}

\author{
Pitchasak CHANKUSON ${ }^{1}$ and Mudtorlep NSSOA $^{1,2, *}$ \\ ${ }^{1}$ Division of Physics, School of Science, Walailak University, Nakhon Si Thammarat 80160, Thailand \\ ${ }^{2}$ Center of Excellence in Plasma Science and Electromagnetic Waves, Walailak University, \\ Nakhon Si Thammarat 80160, Thailand
}

('Corresponding author's e-mail: mnisoa@mail.wu.ac.th)

Received: 21 September 2020, Revised: 3 February 2021, Accepted: 10 February 2021

\begin{abstract}
An electric field in the dielectric barrier electrode system is necessary for ozone production because ozone is produced by the electric discharge of $\mathrm{O}_{2}$ under a high-intensity electric field. The gas discharge plasmas contain energetic particles, such as electrons, ions, atoms, and radicals. The recombination of the $\mathrm{O}$ atom and $\mathrm{O}_{2}$ in the plasma will form $\mathrm{O}_{3}$. In this paper, the dependence of DC electric field formation on electrode geometry and the gap between electrodes and dielectric materials were examined by using computational modeling. Thus, a set of electrode geometry, gap distance, and dielectric material were obtained for high-intensity and uniform electric field generation. The COMSOL Multiphysics software was used for the modeling. Among the electrode geometries of plate-plate, pin-plate and mesh-plate, the mesh-plate generated high-intensity and uniform electric field. In the modeling, dielectric materials, including quartz, mica, alumina, and water, were compared. The highest intensity of electric field occurred on the water surface.
\end{abstract}

Keywords: COMSOL Multiphysics software, Dielectric barrier discharge, High-intensity electric field, Ozone production

\section{Introduction}

The dielectric barrier electrode system is used for studies on gas-insulated high-voltage (HV) systems [1,2] and dielectric barrier discharge (DBD) [3]. It is important for the development of design and analysis of high voltage equipment and various discharge phenomena. The typical problem in these applications is to find the electric field between 2 electrodes. Electric field analysis is necessary for the studies because it leads to dominant phenomena, for instance current termination in an HV system, and acceleration of electron to have enough energy before collisions with atom or molecules, which result in plasma in DBD. Specifically, the DBD has been used in applications, for example ozone generation [3] and cold plasma for medicine [4], food, agriculture [5], and environmental applications [6].

Ozone $\left(\mathrm{O}_{3}\right)$ is a very strong oxidizing agent. It can be produced by 4 processes: corona discharge, DBD, UV radiation, and electrical separation (electrolysis); each method provides high energy to break down the bonds of oxygen atoms in oxygen molecules or water molecules; then, 3 oxygen atoms are merged to form ozone gas. The corona discharge method and the DBD method are commercially available. The DBD method can produce ozone with higher intensity [3] and is a process that can occur at atmospheric pressure or higher. It is popular in the application and the most effective in the production of ozone [7]. For the production of ozone, the DBD method consists of an ozone tube (ozonizer) air preparation system and an electric power supply. The ozone tube has a structure consisting of 2 
http://wjst.wu.ac.th

electrodes. It is a parallel sheet or cylindrical type with double axes and with dielectric material in the middle. Generally, borosilicate glass is used as a dielectric material.

Ozone tubes create a high-intensity electric field and a plasma. In the plasma, there are collisions between electrons and atoms or molecules at all times. With collisions between electrons and oxygen molecules, molecules will have higher energy. If the electrons have at least 6.0 or $8.4 \mathrm{eV}$ energy, the oxygen molecules will be stimulated to have $A^{3} \Sigma_{u}^{+}$or $B^{3} \Sigma_{u}^{-}$, respectively. The $2^{\text {nd }}$ is unstable and makes the oxygen molecules break down to get 2 oxygen atoms. The combination of oxygen atoms and oxygen molecules results in ozone production as in reactions (R1) and (R2).

$$
\begin{aligned}
& e+O_{2} \rightarrow e+O_{2}\left(A^{3} \Sigma_{u}^{+}\right) \rightarrow e+O\left({ }^{3} P\right)+O\left({ }^{3} P\right) \rightarrow O_{2}+O\left({ }^{3} P\right) \rightarrow O_{3} . \\
& e+O_{2} \rightarrow e+O_{2}\left(B^{3} \Sigma_{u}^{-}\right) \rightarrow e+O\left({ }^{3} P\right)+O\left({ }^{1} D\right) \rightarrow O_{2}+O\left({ }^{3} P\right) \rightarrow O_{3} .
\end{aligned}
$$

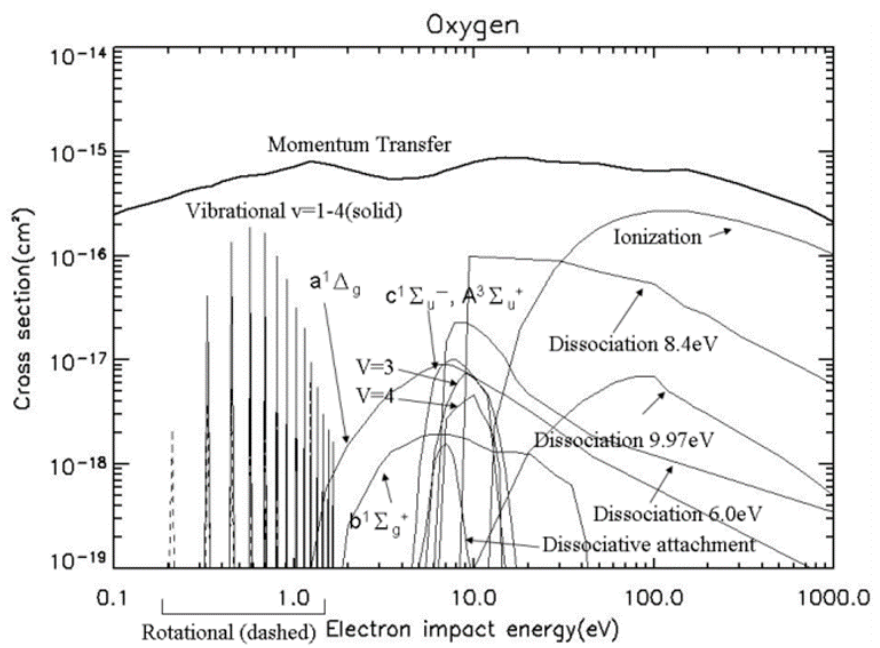

Figure 1 Cross-section of oxygen.

The development of ozone tubes for increased efficiency can be done by designing the characteristics of the electrode and choosing a suitable dielectric material. To obtain a high-intensity electric field that can lead to higher intensity ozone [8], ozone tubes should have features such as coils [9], screw [10], and needle with different terminal tips in the ozone tube [11], etc. High-intensity electric fields are important in the formation of ozone. This is because they produce electrons with enough energy to form plasmas, as well as the number of electrons required. Thus, more oxygen atoms and a chance of ozone with higher intensity and higher production efficiency are created. The high-intensity electric field is derived from the design of various parts such as the features of ozone tube, power supply, etc.

\section{Plasma gas discharge}

Plasma generation by gas discharge can be accomplished by supplying the electric voltage $V$ to the cathode and anode, which are spaced apart by $d$ within the gas container. The electrical field $\vec{E}$ will accelerate the free electrons until they have enough energy to hit the gas atoms and then break down (ionization) continuously and quickly until the gas discharge occurs through the process called electric discharge. The resulting electric discharge process can be explained as follows:

When a cosmic ray hits the cathode, free electrons of $N_{0}$ will be produced at the cathode, which is accelerated by the electric field $\vec{E}$ moving to the anode. While moving, a collision with the gas atoms 
causes ionization. The positive ion and the free electrons continuously increase. Therefore, the number of all electrons moving to the anode is,

$$
N_{t}=\frac{N_{0} e^{\alpha d}}{1-\gamma\left(e^{\alpha d}-1\right)}
$$

where $\alpha, \gamma$ are the Townsend ionization coefficient and secondary electron emission coefficient of the cathode. If $\gamma\left(e^{\alpha d}-1\right)=1$ causes the ionization of the atomic gas at an avalanche (avalanche discharge) up, self-sustaining, it leads to gas breakdowns between the electrode, a condition known as, Townsend's breakdown criterion or self-sustaining discharge criterion, which is a theory that describes the gas discharge proposed by Townsend, and called the Townsend mechanism. According to the Townsend experiment,

$\alpha=A p e^{-\frac{B p}{E}}$ where, $E_{b}$ is the electric field causing the breakdown. Substitute the value of $\alpha$ to get:

$$
E_{b}=\frac{B p}{\ln A p d-\ln \left[\ln \left(\frac{1}{\gamma}-1\right)\right]}
$$

Where $A, B$ are the constant values of each type of gas and $p$ is the pressure of the gas in torr units, in the case of air $A=15 \mathrm{~cm}^{-1} \operatorname{torr}^{-1}, B=365 \mathrm{~V} \mathrm{~cm}^{-1} \operatorname{torr}^{-1}$ and $\gamma=0.01$ [12]. From Eq. (2), the breakdown voltage $V_{b}$ can be obtained from $V_{b}=E_{b} d$. When considering the pressure at 760 torr, the graph shows the change of $E_{b}$ and $V_{b}$ at $d$ as shown in Figures 2(a) and 1(b), respectively, it is found that $E_{b}$ decreases as $d$ increases, and reaches $30 \mathrm{kV} / \mathrm{cm}$ when $d>1 \mathrm{~cm}$, while the Paschen curve $V_{b}(d)$ will have a minimum $V_{b}$ of about $300 \mathrm{~V}$ at a distance of about $10 \mu \mathrm{m}$ [13].

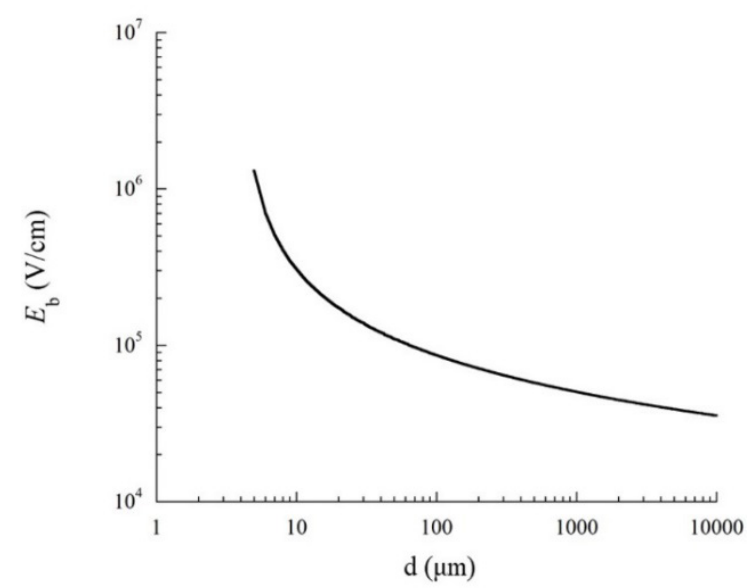

(a)

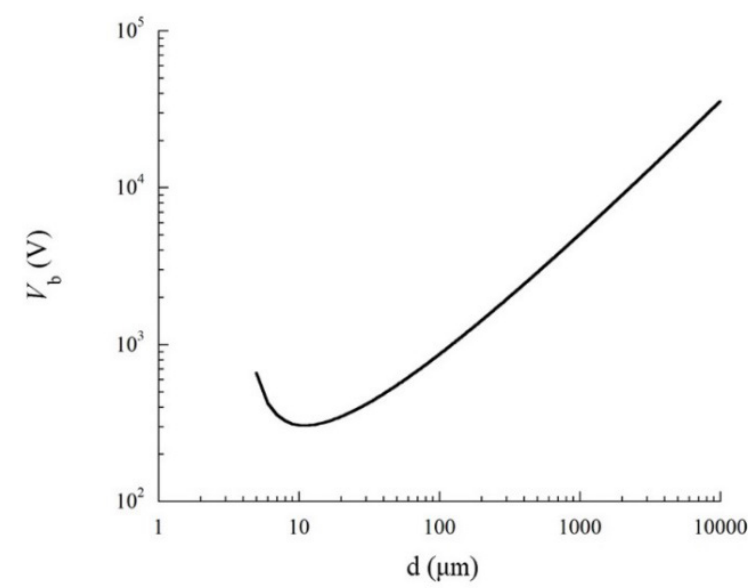

(b)

Figure 2 The electric field (a) and potential difference (b) for the breakdown at any $d$ of air at a pressure of 760 torr. 
http://wjst.wu.ac.th

\section{Electric field simulation}

Too develop an electrode that generates uniform and high-intensity electric fields which will cause a gas discharge to form a large amount of plasma, various configurations of electrodes were considered. For an ideal electrode, a parallel plate with an infinite size, the electric field between the electrode plates can be obtained analytically. But, for electrodes with a limited size, asymmetrical and irregular shape, the resulting electric field is in the form of a non-linear partial differential equation. Numerical methods must be used to determine the distribution of the electric field. Currently, this can be done using simulation software such as COMSOL Multiphysics, MATLAB/Simulink, which is the software developed to solve numerical problems using methods such as Finite element method (FEM) and Finite volume or Finite difference methods (FDM) [14]. For example, Kara et al. [15] used COMSOL software that uses FEM in calculating electric field and electric potential from the model of rod-tip and plate-type electrode in their study on the effect of dielectric materials on electric field values in the air gap by showing that the maximum electric field intensity occurs at the ends of the rods and increases with increase in the dielectric thickness or use of materials with high relative dielectric constant, reducing the distance between the electrode. Besides, Foruzan et al. [16] used COMSOL software to simulate needle-to-plate and circular-to-sheet electrodes in their study on the distribution of electric fields in the air gap between the electrode and when changing the distance between the electrode and dielectric. The simulation results showed that the needle and sheet electrodes have an uneven distribution of the electric field (nonuniform), while the spherical and plate electrodes have a semi-uniform electric field distribution (semiuniform). From this study, computational simulation is useful in studies on design and testing, to find the suitable electrode configuration.

The objective of this research is to use COMSOL Multiphysics software to simulate timeindependent electric field generation by using the parallel plate electrode to study the effect of change in the distance between the electrodes and type of dielectric materials, and the models at the top of the electrodes are pin, line, and mesh for study on the shape of the electrode. We focus on generating an electric field for accelerating electrons until electrons have high energy and move to collision oxygen molecules. Molecules break down to get 2 oxygen atoms. These oxygen atoms are a substrate for ozone formation according to the reaction (R1) and (R2). The simulation results will be used to design the ozone tube which causes high-intensity and uniform electric fields for high-efficiency ozone production. However, ozone production may be to depend on other conditions such as temperature, reaction rate, etc., which will need further studies in the future.

\section{Simulation methods and results}

In this research, models were formed based on various conditions, and partial boundary differential equations were solved to determine the intensity and distribution of electric fields using COMSOL Multiphysics software with the following steps:

1) Selection of the module used in the study: In this research, the AC/DC module which determines the equation was used.

2) Creation of the geometry of the model to be studied: In this research, there are parallel-plate electrode, pin-plate, line-plate, and mesh-plate with dielectric material between the electrodes. By placing on the bottom electrode, the size of the electrode, distance between terminals and the type of dielectric materials are determined.

3) Determination of the electrical properties of the electrode to be a perfect conductor and determination of the constant value of the dielectric material which can select the relative dielectric constant $\varepsilon_{r}$ of each material

4) Determination of the size of the elements by dividing the model into small pieces called elements. Each element is linked to a mesh. The connection points in each element are called nodes, where the solutions are obtained. In this research, the mesh size of extremely fine was used.

5) Running each model to obtain the electric field $\vec{E}(r)$, which can be shown in 1 , 2 , or 3 dimensions. 
http://wjst.wu.ac.th

In this study, to investigate the effect of distance between electrodes and dielectric materials on the formation of high intensity electric field, parallel plate electrode simulation was carried out first. The $2^{\text {nd }}$ part was the study on the effect of electrode geometries on the uniformity and high-intensity of electric field with details as follows:

\section{Study on the effects of distance between electrodes}

The model of the electrodes consists of two parallel circular plates. Each plate has a thickness of 0.5 $\mathrm{mm}$ and a radius of $20 \mathrm{~cm}$. The two electrodes are spaced $\mathrm{d}_{2}$ apart, with quartz of thickness $\mathrm{d}_{1}$, as dielectric material, placed on the bottom electrode. There is an air gap between the top plate electrode and the dielectric surface of $d=d_{2}-d_{1}$ as shown in Figure 3. When the DC potential gives $V=1,000 \mathrm{~V}$ between the 2 electrodes and the electric field $\vec{E}$ within the gap is calculated, then $d$ is $0-5,000 \mu \mathrm{m}$.

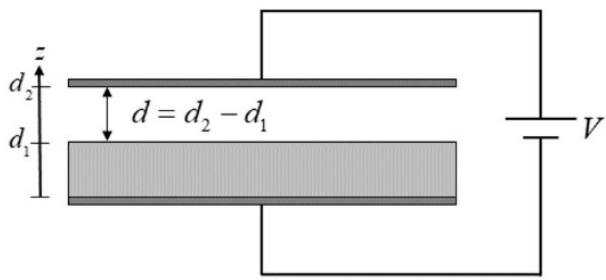

Figure 3 Characteristics of the electrode used in the model which consists of 2 circular conductor plates and a dielectric sheet placed on the bottom terminal with dielectric thickness $d_{1}=0.5 \mathrm{~cm}$ and gaps $d=d_{2}-d_{1}$.

The simulation results are shown in Figure 4(a). It is shown that the electric field at a distance less than $0.06 \mu \mathrm{m}$ has a constant equal to $2 \times 10^{5} \mathrm{~V} / \mathrm{m}$, which is the value of the electric field when there are no dielectric materials (dotted line). When the gap distance is too small, the electric field inside the gap is equal to the electric field inside the dielectric material, similar to the electric field between the electrodes in the absence of dielectric material, as shown in Figure 5. At $d=0.06 \mu \mathrm{m}$ the electric field increases sharply to $8.40 \times 10^{5} \mathrm{~V} / \mathrm{m}$ or 4.2 times because of the bounded charge which occurs at the surface of the dielectric material from polarization within the dielectric material induced by an applied electric field. In a range of about $0.06<d<100 \mu \mathrm{m}$ the electric field is highest and constant. Then, the electric field decreases as $d$ increases as shown in Figure 4(a), where the electric field is approximately $8.40 \times 10^{5}$ $\mathrm{V} / \mathrm{m}$ when $0.06<d<100 \mu \mathrm{m}$ and will be less than $2 \times 10^{5} \mathrm{~V} / \mathrm{m}$ when $d>5 \mu \mathrm{m}$ as shown- in Figure 4(b). 


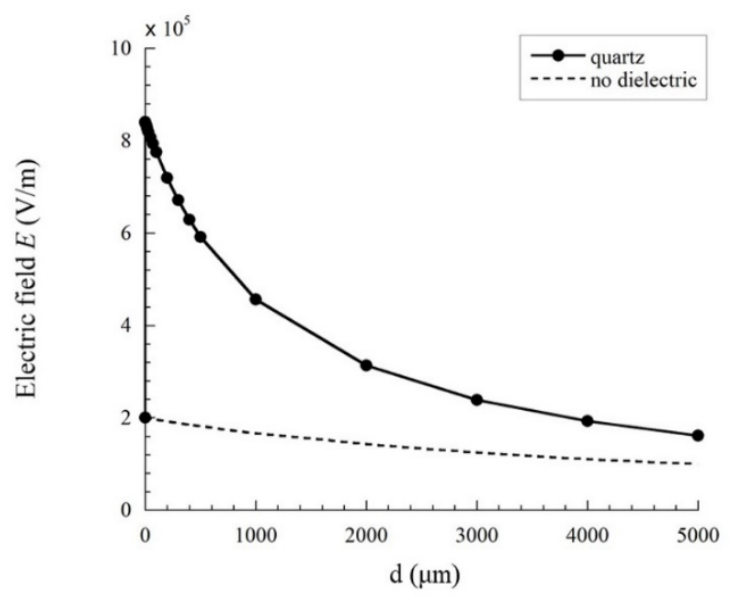

(a)

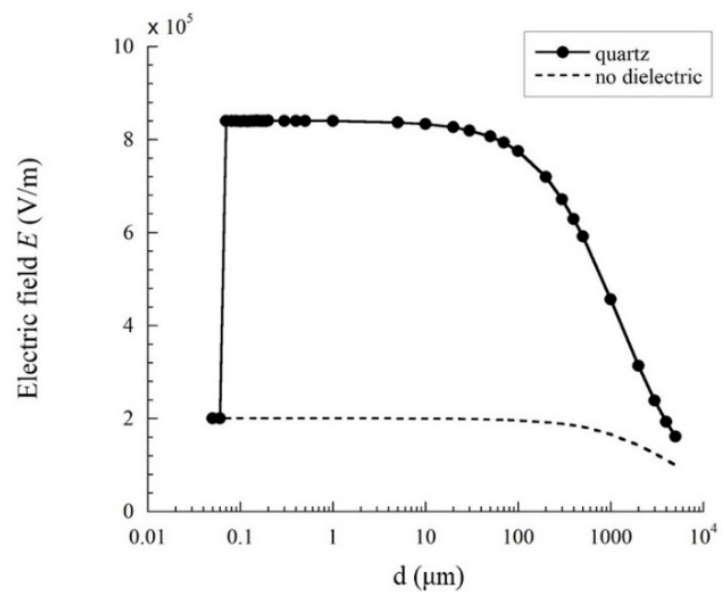

(b)

Figure 4 The electric field inside the air gap between the parallel plate electrode plotted in linear axis (a) and semi-log axis (b) by providing a potential voltage of $1,000 \mathrm{~V}$ when using quartz as dielectric and without dielectric materials at the center position of the electrodes, where $x=0$ and $y=0$.

\section{Study on the effects of dielectric materials}

As shown in Figure 3, using the model consisting of 4 kinds of dielectric materials, quartz, mica, alumina, and water, with thicknesses of $0.5 \mathrm{~cm}$, the gap between the top electrode and the dielectric surface $d=100 \mu \mathrm{m}$ provides a $1,000 \mathrm{~V}$ direct current voltage between the 2 electrodes. The electric field in the dielectric material ( $z=0-5,000 \mu \mathrm{m}$ ) and the gap $(5,000<z \leq 5,100 \mu \mathrm{m})$ are determined when each dielectric material is used.

From Figure 5, when there is a dielectric material between the electrode, an electric field is created within the material which is less than the electric field between the electrode without dielectric, in which water is the least valuable and quartz the most valuable. However, the electric field within the gap between the electrodes when there are dielectric materials will be higher when there are no dielectrics.

In the case of dielectric, water is the dielectric that has the highest electric field, which is 30 times higher than in the absence of dielectric, followed by alumina, mica, and quartz which are 8, 6 , and 4 times higher than in the absence of dielectric, respectively, when considered together. With the relative dielectric constant $\left(\varepsilon_{r}\right)$ of the material shown in Table 1, it is found that the electric field within the gap increases when dielectric with increasing $\varepsilon_{r}$ is used.

Table 1 Maximum electric field in the gap between parallel sheet electrodes with different types of dielectrics.

\begin{tabular}{lcc}
\hline Electric material & Comparative dielectric constant $\left(\varepsilon_{r}\right)$ & Maximum electric field (V/m) \\
\hline Quartz & 4.3 & $7.75 \times 10^{5}$ \\
Mica & 6 & $1.07 \times 10^{6}$ \\
Alumina & 9.5 & $1.57 \times 10^{6}$ \\
Water $\left(20^{\circ} \mathrm{C}\right)$ & 81 & $6.18 \times 10^{6}$ \\
\hline
\end{tabular}




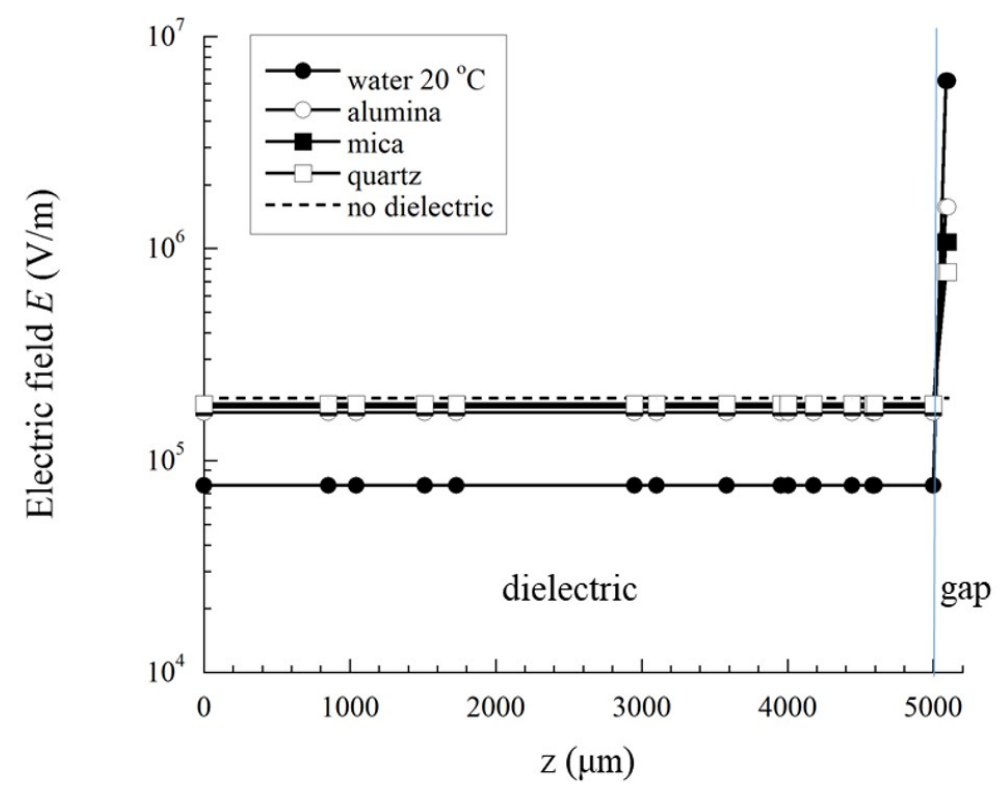

Figure 5 The electric field between the parallel plates in the middle of the plate within the electric material $(0 \leq z \leq 5,000 \mu \mathrm{m})$ and inside the gap $(5,000<z \leq 5,100 \mu \mathrm{m})$, applied voltage $1,000 \mathrm{~V}$.

\section{Study on the effects of electrode geometries}

From the model in Figure 3, the top electrode is changed to pin, line, and mesh sizes of $1 \mathrm{~cm}^{2}$ as shown in Figures 6(a), 6(b), and 6(c), respectively. The electrode is a square of $20 \times 20 \mathrm{~cm}^{2}$, the distance between the electrode and the dielectric surface (gap width) $d=100 \mu \mathrm{m}$. When supplying the DC voltage $V=1,000 \mathrm{~V}$, the electric field is calculated in the gap when using different types of electrodes.

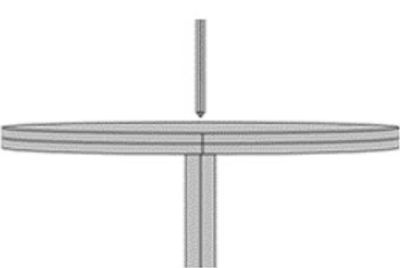

(a)

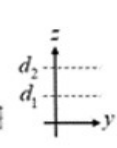

$y$

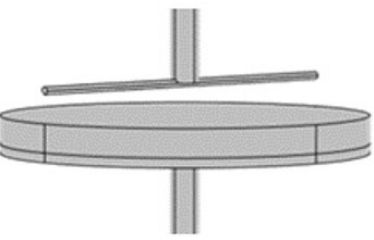

(b)

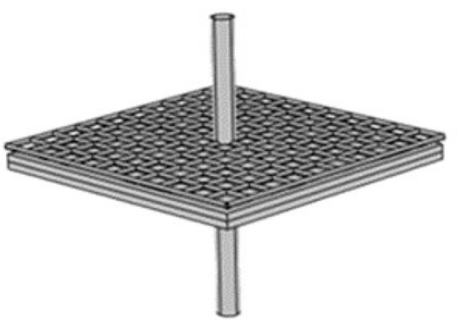

(c)

Figure 6 Model of the electrode consisting of the top terminal (a) pin (b) line and (c) mesh. The lower electrode is covered by quartz as dielectric, where $d_{1}=0.5 \mathrm{~cm}$ and $d=d_{2}-d_{1}=100 \mu \mathrm{m}$.

The electric field in the $\mathrm{z}$-axis between the electrode and the surface of the dielectric material at the center point $x=0$ and $y=0$ when using the 4 electrode types, are shown in Figure 7 . It is found that the pin, line, and mesh electrodes have higher an electrical field than the plate electrode at all $z$ positions. The electric field of the pin electrode has the highest values and the highest linear increase rates 
http://wjst.wu.ac.th

as well when the pin position ( $z=d=100 \mu \mathrm{m}$ ) has the maximum electric field of $6.98 \times 10^{6} \mathrm{~V} / \mathrm{m}$; while the electric field of the sheet electrode has a constant of $7.75 \times 10^{5} \mathrm{~V} / \mathrm{m}$, and the electric field of the mesh electrode is relatively constant and approximately double the electric field of the sheet electrode, as shown in Table 2.

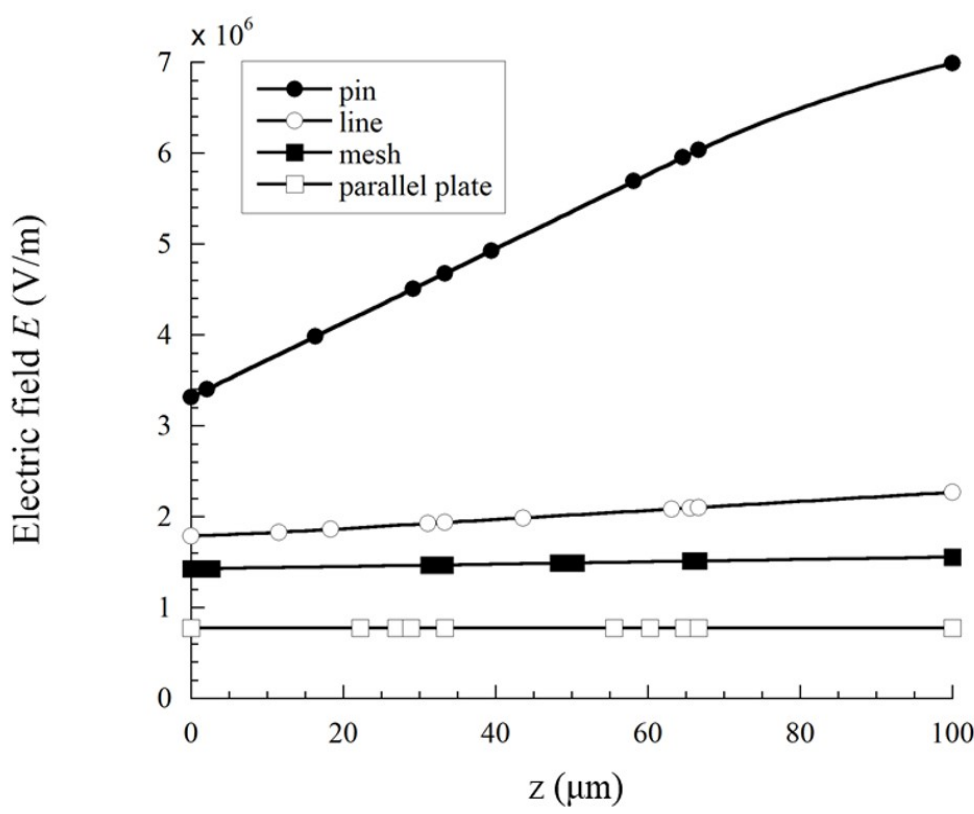

Figure 7 The electric field inside the air gap in the z-axis and $z=0, y=0$, when $d=100 \mu \mathrm{m}$ when using 4 electrode types, quartz was used as the dielectric material, applied voltage 1,000 V.

Table 2 The maximum electric field within the air gap along the z-axis and $x=0, y=0$ when $d=100$ $\mu \mathrm{m}$ of different electrodes when quartz was used as a dielectric material.

\begin{tabular}{lc}
\hline Electrodes & Maximum electric field along the axis $\mathbf{z}(\mathbf{V} / \mathbf{m})$ \\
\hline Parallel plate & $7.75 \times 10^{5}$ \\
Pin-plate & $6.98 \times 10^{6}$ \\
Line-plate & $2.26 \times 10^{6}$ \\
Mesh-plate & $1.55 \times 10^{6}$ \\
\hline
\end{tabular}

In Figure 8 the two-dimensional distribution of the electric field on the $x y$ plane at the position $z$ $=100 \mu \mathrm{m}$ is presented. The figure shows that the electric fields of the pin and line electrodes are localized at the electrodes, they will decrease to zero when only $1 \mathrm{~cm}$ far from the electrons as shown in Figures 8(a) and 8(b), respectively. While the electric field of the mesh electrode in Figure 8(c) will be uniform throughout the area of the electrode of $20 \times 20 \mathrm{~cm}^{2}$. The difference in the electric field not more than 10 times and without the position in which the electric field is zero. 


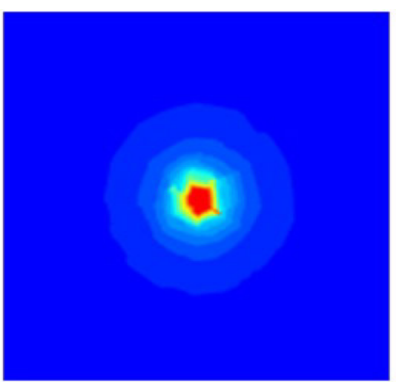

(a)

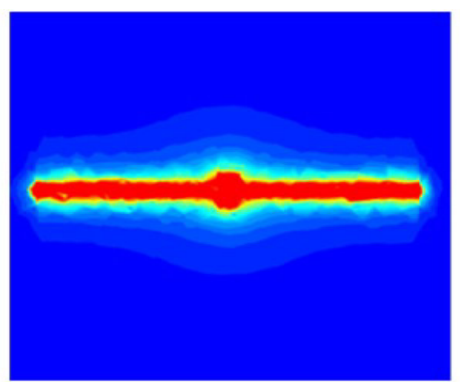

(b)
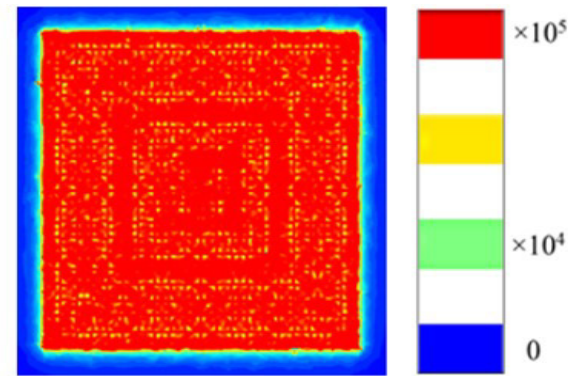

(c)

Figure 8 Electric field $\vec{E}$ on the $x y$ plane where $z=100 \mu \mathrm{m}$ of the (a) pin (b) line and (c) mesh. The electric field is represented by the scale of blue color, the electric field is zero and the red electric field has the highest value, $10^{5} \mathrm{~V} / \mathrm{m}$, applied voltage $1,000 \mathrm{~V}$.

\section{Study on the effect of mesh size}

From the models in Figure 6(c), there is a change in the mesh size from 3 to 2 and $1 \mathrm{~cm}^{2}$, with the distance between the top electrode and the dielectric surface $d=100 \mu \mathrm{m}$ when providing a DC voltage of $1,000 \mathrm{~V}$ between both electrodes, calculating the electric field in the gap when using each mesh size.

The electric field on the $x y$ plane at $z=100 \mu \mathrm{m}$ when using meshes of 3,2 , and $1 \mathrm{~cm}^{2}$ with characteristics shown in Figures 9(a), 9(b) and 9(c), respectively. It is shown that the electric field becomes more uniform when the mesh size was decreased. When the mesh is $2 \mathrm{~cm}^{2}$ the area where the electric field is greater than $10^{5} \mathrm{~V} / \mathrm{m}$ (red) has an area of more than $50 \%$ and when the mesh size is reduced to $1 \mathrm{~cm}^{2}$, there will be an area where the electric field is greater than $10^{5} \mathrm{~V} / \mathrm{m}$ more than $90 \%$.

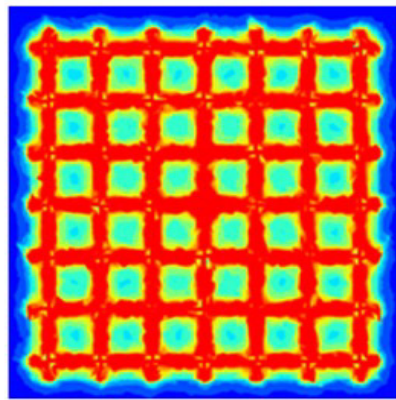

(a)

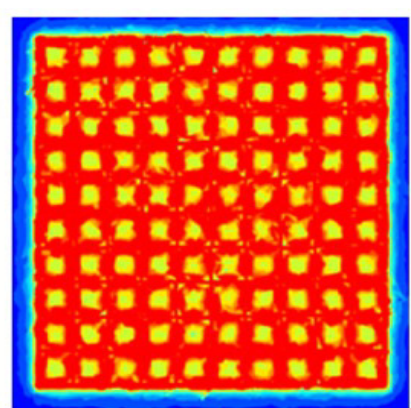

(b)
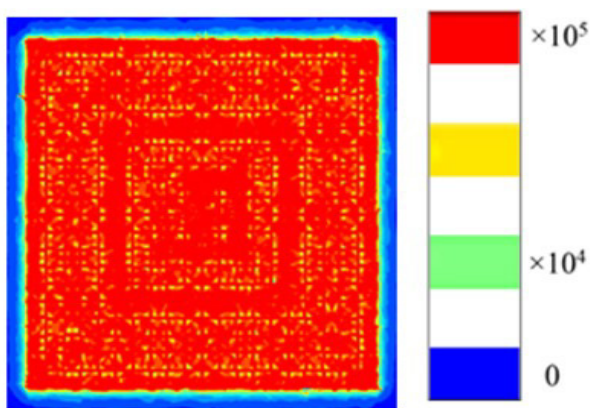

(c)

Figure 9 Electric field $\vec{E}$ on the $x y$ plane, where $z=100 \mu \mathrm{m}$ for the mesh size of (a) $3 \mathrm{~cm}^{2}$ (b) $2 \mathrm{~cm}^{2}$ and (c) $1 \mathrm{~cm}^{2}$. The electric field is represented by the scale of blue color, the electric field is zero, the red electric field is higher than $10^{5} \mathrm{~V} / \mathrm{m}$, applied voltage $1,000 \mathrm{~V}$.

\section{Discussion}

From the simulation results obtained from this study on the electric field between electrodes when there is a DC electric potential between the electrodes, it was found that the electric field in the air gap depends on the distance between the electrodes dielectric material and the shape of the electrode. It was also found that when using a parallel plate with a dielectric placed on the bottom electrode, the electric field within the gap increases sharply as compared to the electric field in the absence of dielectrics. The 
http://wjst.wu.ac.th

increase is approximately $30,8,6$, and 4 times when water, alumina, mica, and quartz are respectively used as dielectric materials. The electric field is the maximum electric field within the gap at $d$ in the range of $0.06<d<100 \mu \mathrm{m}$. The electric field in the gap near the surface of the dielectrics increases due to the bounded charge $\left(\sigma_{b}\right)$ which occurs at the surface of the dielectric material by $\sigma_{b}=-\vec{\nabla} \cdot \vec{P} \propto P$, where $P$ is the polarization within the dielectric material induced by an electric field.

To obtain a maximum electric field between the electrodes while $V, \varepsilon$ and $d$ are constant, the shape of the upper electrode are varied. When a pin electrode is used, it has the electric field with the highest intensity but has the highest non-uniformity. When the distance from the pin is greater than $1 \mathrm{~cm}$, the electric field disappears. The use of a $1 \mathrm{~cm}^{2}$ mesh electrode provide an electric field with 2 times higher intensity than the sheet electrode, and the electric field is uniform throughout the electrode with an area of $20 \times 20 \mathrm{~cm}^{2}$. Therefore, the mesh electrode is suitable to use for high-efficiency ozone tubes. If the width of the gap is chosen as $d=100 \mu \mathrm{m}$, to have a discharge and produce ozone at atmospheric pressure, an electric field greater than $8.64 \times 10^{6} \mathrm{~V} / \mathrm{m}$ is required. Therefore $V>0.9,3,4.4$ and $6 \mathrm{kV}$ have to supplied when using water, alumina, mica, and quartz, respectively, as shown in Figure 10.

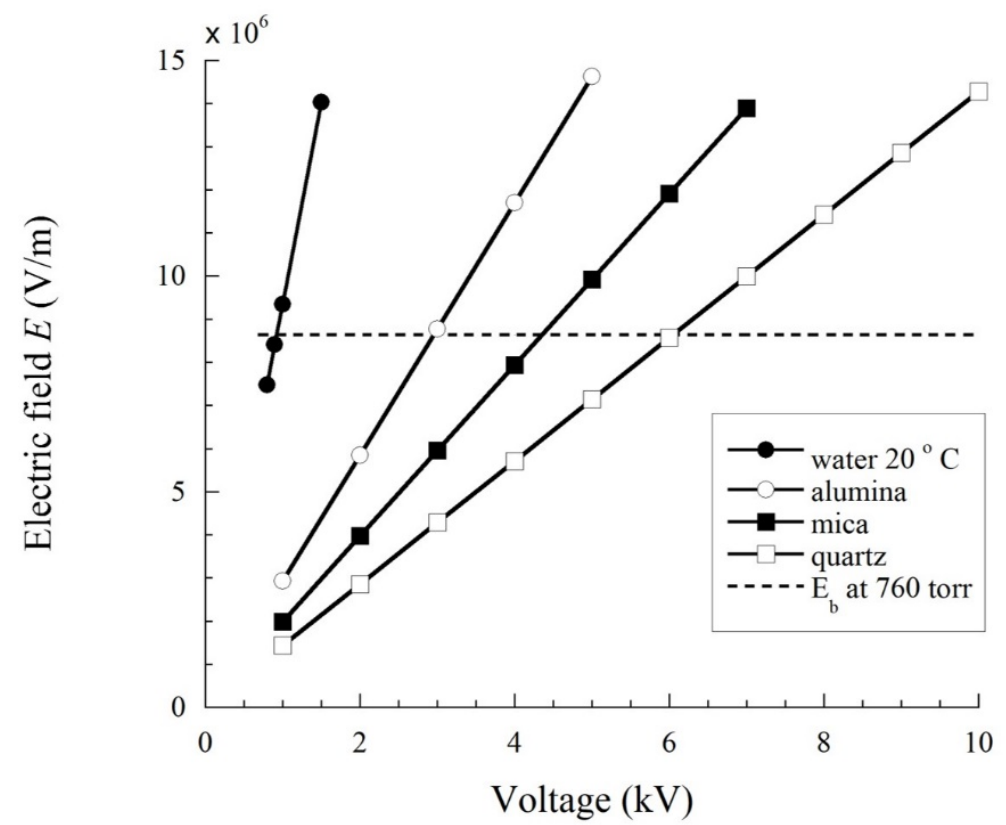

Figure 10 Electric field and minimum electric potential for breaking down at a pressure of 760 torr when using various electric materials for polarized mesh with the mesh size of $1 \mathrm{~cm}^{2}$.

\section{Conclusions}

For typical parallel plate geometry, the gap less than about $100 \mu \mathrm{m}$ give constant and maximum electric field value, after that the electric field decrease as increase gap distance. Furthermore, the addition of a dielectric material between the electrodes when the air gap is less than $100 \mu \mathrm{m}$ can increase the electric field in the gap many times, which was directly proportional with the dielectric constant. Finally, to obtain a more uniform electric field of more than $90 \%$, an electrode with a mesh size of less than $1 \mathrm{~cm}^{2}$ is required. The electric field from this simulation is a local field from all discharge areas of the ozone tube. For using can scale up it from this local electric field. The use of suitable dielectric materials can also reduce the applied voltage for the gas breakdown for ozone production. 
http://wjst.wu.ac.th

\section{Acknowledgements}

This research was funded by the Research and Researchers for Industries (RRI) by the Thailand Research Fund with Contract No. PHD61I0029. The authors also thanked the Center of Excellence in Plasma and Electromagnetic Waves, Walailak University, Thailand for facilitating the sources and software packages and Nakhon Si Thammarat Rajabhat University, Thailand for permitting full-time leave for conducting this study.

\section{References}

[1] M Sjoberg, YV Serdyuk, SM Gubanski, and MAS Leijon. Experimental study and numerical modelling of a dielectric barrier discharge in hybrid air-dielectric insulation. J. Electrostat. 2003; 59, 87-113.

[2] M Talaat. Electrostatic field calculation in air gaps with a transverse layer of dielectric barrier. $J$. Electrostat. 2014; 72, $422-7$.

[3] R Brandenburg. Dielectric barrier discharges: Progress on plasma sources and on the understanding of regrimes and single filaments. Plasma Sources Sci. Technol. 2017; 26, 053001.

[4] G Isbary, T Shimizu, YF Li, W Stolz, HM Thomas, GE Morfill and JL Zimmermann. Cold atmospheric plasma devices for medical issues. Expert Rev. Med. Devices 2013; 10, 367-77.

[5] NN Misra, OK Schluter and PJ Cullen. Cold plasma in food and agriculture. Academic Press, Elsevier Inc., 2016.

[6] M Magureanu, C Bradu and VI Parvulescu. Plasma processes for the treatment of water contaminated with harmful organic compounds. J. Phys. D Appl. Phys. 2018; 51, 313002.

[7] S Pekárek. Experimental study of surface dielectric barrier discharge in air and its ozone production. J. Phys. D Appl. Phys. 2012; 45, 075201.

[8] R Brandenburg. Dielectric barrier discharges: Progress on plasma sources and on the understanding of regimes and single filaments. Plasma Sources Sci. Technol. 2017; 26, 053001.

[9] S Radautsan and G Parissakis. Scientific and technological achievements related to the development of European cities. Springer-Science \& Business Media, 2013.

[10] T Fujishima, T Kawaguchi, T Amano and T Yamashita. Ozone generation properties of screw-type electrode ozonizer by divided outer electrodes. In: Proceedings of the $3^{\text {rd }}$ International Conference on Electric and Electronics, Nagasaki, Japan. 2013, p. 311-4.

[11] M Jongsuphaphong, S Sirianuntapiboon and B Limmeechokchai. Evaluation of a pilot scale high pressure plasma ozonizer for use in wastewater treatment. Afr. J. Biotechnol. 2010; 9, 5151-61.

[12] A Fridman. Plasma chemistry. Cambridge University Press, USA, 2011.

[13] DB Go and DA Pohlman. A mathematical model of the modified Paschen's curve for breakdown in microscale gaps. J. Aappl. Phys. 2010; 107, 103303.

[14] T Defraeye. Advanced computational modelling for drying processes: A review. Appl. Energ. 2014; 131, 323-44.

[15] A Kara, O Kalenderli and K Mardikyan. Effect of dielectric barriers to the electric field of rod-plane air gap. In: Proceedings of the COMSOL Users Conference, Prague, Czech Republic. 2006.

[16] E Foruzan, AAS Akmal, K Niayesh, J Lin and DD Sharma. Comparative study on various dielectric barriers and their effect on breakdown voltage. High Volt 2018; 3, 51-9. 